This is a difficult clinical problem, and it will remain largely a matter of experience to determine if and when surgical exploration of the abdomen is performed on patients presenting with continued unexplained fever. Any clue to the possible existence of an intra-abdominal lesion, even a vague one, can be extremely helpful in reaching this decision. There will still be a few patients in whom there are no obvious clues but who may still require surgical exploration. Perhaps these patients might be best served by the combination of a surgically minded physician and a conservative surgeon.

1 Ben-Shoshan, M. Gius, J. A., and Smith, I. M., Surgery, Gynecology and Obstetrics, 1971, 132, 994.

\section{Volvulus of the Stomach}

Most viscera have a single blood supply which runs in the mesentery, so that volvulus of the organ rapidly imperils the blood supply and produces gangrene. The stomach is an exception, for its blood supply is so rich and varied that volvulus usually presents as a chronic disorder. Acute gastric volvulus leading to gangrene is a rarity, and most surgeons will deal only with one or two such patients in a professional lifetime.

There are two main varieties of gastric volvulus. The first is organo-axial, which is rotation round the long axis of the stomach (the line between pylorus and cardia). The second is mesenterio-axial, which is the rotation of the stomach from right to left or left to right about the long axis of the gastro-hepatic ligament (an imaginary line drawn through the mid lesser curve and mid greater curve). In a recent review of published reports $59 \%$ of cases were found to be organo-axial and $29 \%$ mesentero-axial; the remainder were either mixed or unclassified. ${ }^{1}$ The sexes are equally affected, and the condition is commonest in the fifth decade of life.

For volvulus to occur there must be some ligamentous laxity, for the stomach is held at its oesophageal end by the ligaments round the hiatus and at its distal end by the descending part of the duodenum becoming retroperitoneal. There is usually an obvious predisposing cause, the commonest being a para-oesophageal hiatus hernia. In this condition the stomach rolls upwards into the posterior mediastinum, thus producing an organo-axial volvulus. Though the whole stomach may lie upside down in the chest, the condition may be virtually symptomless. Other diaphragmatic hernias resulting from trauma or congenital defects may occasionally be the underlying cause.

Eventration of the left diaphragm is now recognized as an important predisposing cause. ${ }^{2}$ The large space beneath the left cupola of the diaphragm is filled by the colon, which in turn pulls up the greater curve of the stomach, causing organo-axial volvulus. Other predisposing causes are pyloric or duodenal obstruction, which produces chronic dilatation of the stomach. It is suggested that a heavy meal may also predispose to the condition by approximating the pylorus and cardia.

Acute volvulus presents as an emergency with severe upper abdominal pain, which is accompanied by three important features-Borchadt's triad. They are, firstly, initial vomiting followed by retching and an inability to vomit; secondly, epigastric distension; and, thirdly, the inability to pass a nasogastric tube. Attempts to investigate the patient by barium or other contrast medium show complete obstruction at the lower end of the oesophagus. Urgent exploration is required, otherwise gangrene of the stomach is likely to necessitate resection. The mortality rate of the condition has been estimated at $30-40 \% .^{2}$

Chronic recurrent volvulus is the commoner variety, though it is still an unusual condition. It is difficult to diagnose clinically, but the patient usually gives a history of attacks of epigastric pain associated with a feeling of distension, and despite eructation there is no relief of the symptoms. The diagnosis becomes apparent on barium examination, when characteristic distortion of the stomach is seen. Many patients have only minimal symptoms and require no treatment. When the symptoms are disabling, surgical treatment should be directed towards removing the cause when this is possible. Para-oesophageal or other diaphragmatic hernias are dealt with by reduction and repair. Relief of pyloric or duodenal obstruction is needed if present. For those patients with eventration of the diaphragm various methods of treatment such as gastropexy, partial gastrectomy, anastomosis of the gastric antrum to the fundus, and gastrojejunostomy have all been tried with different degrees of success. The most logical and most successful operation is the so-called colonic displacement of the stomach. ${ }^{2}$ In this procedure the greater curve of the stomach is completely freed from the colon so that the transverse colon and great omentum can roll up under the left diaphragm without dragging the stomach after them. A gastropexy is added after this procedure. At long-term follow up 12 out of 14 patients showed a successful result after this procedure. ${ }^{2}$

1 Wastell, C., and Ellis, H., British fournal of Surgery, 1971, 58, 557. 2 Tanner, N. C., American fournal of Surgery, 1968, 115, 505.

\section{Colleagues in Health Care}

One of the best concepts to emerge from general practice in the last few years has been that of the health team, comprising not only doctors and nurses but social workers, health visitors, medical secretaries, and practice managers. Nevertheless, descriptions of the part played by these invaluable colleagues have almost always included one comment: that at the start of an attachment scheme doctors had relatively little idea of either the qualifications for or the proper role of the particular discipline. And for their part the other members of the practice team have sometimes complained of initial misunderstandings, particularly in the early days ("the majority of doctors ... . were totally alienated from the health visitors, and . . . attitudes ranged from indifference to hostility"). ${ }^{1}$

This week (p. 479) we start a new series by a special correspondent on "Colleagues in Health Care". This will inciude descriptions of the qualifications and training of a variety of people, both in the domiciliary health team and outside it. We hope that at a time when the health services are moving towards a closer integration these will seem apposite.

1 Hockey, L., Feeling the Pulse. London, Queen's Institute of District Nursing, 1966. See B.M.7. 1966, 2, 1609. 